\title{
Leukoaraiosis May Confound the Interpretation of CT Perfusion in Patients Treated with Mechanical Thrombectomy for Acute Ischemic Stroke
}

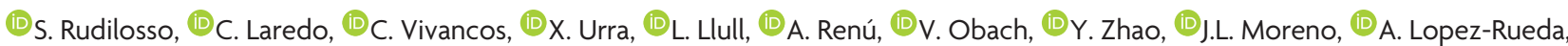 \\ (1)S. Amaro, and ํㅗ. Chamorro

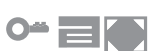

\begin{abstract}
BACKGROUND AND PURPOSE: Leukoaraiosis frequently coexists in patients with acute stroke. We studied whether leukoaraiosis could confound the interpretation of CTP findings in patients treated with mechanical thrombectomy.

MATERIALS AND METHODS: We analyzed 236 patients with stroke treated with mechanical thrombectomy and studied with CTP, of whom 127 (53.8\%) achieved complete reperfusion. Periventricular white matter hyperintensities on MR imaging and hypodensities on NCCT were assessed through the Fazekas score. CTP-predicted nonviable tissue was defined as relative CBF $<30 \%$, and final infarct volume was quantified in DWI. We estimated mean MTT, CBV, and CBF in the asymptomatic hemisphere. In patients achieving complete reperfusion, we assessed the accuracy of nonviable tissue to predict final infarct volume using the intraclass correlation coefficient across periventricular hyperintensity/hypodensity Fazekas scores and variable relative CBF cutoffs.
\end{abstract}

RESULTS: MTT was longer (Spearman $\rho=0.279, P<.001$ ) and CBF was lower $(\rho=-0.263, P<.001$ ) as the periventricular hyperintensity Fazekas score increased, while CBV was similar across groups ( $\rho=-0.043, P=.513$ ). In the subgroup of patients achieving complete reperfusion, nonviable tissue-final infarct volume reliability was excellent in patients with periventricular hyperintensity Fazekas score grade 0 (intraclass correlation coefficient, $0.900 ; 95 \% \mathrm{Cl}, 0.805-0.950$ ), fair in patients with periventricular hyperintensity Fazekas scores 1 (intraclass correlation coefficient, $0.569 ; 95 \% \mathrm{Cl}, 0.327-0.741$ ) and 2 (intraclass correlation coefficient, $0.444 ; 95 \% \mathrm{Cl}, 0.165-0.657$ ), and poor in patients with periventricular hyperintensity Fazekas score 3 (intraclass correlation coefficient, $0.310 ; 95 \% \mathrm{Cl},-0.359-0.769$ ). The most accurate cutoffs were relative $\mathrm{CBF}<30 \%$ for periventricular hyperintensity Fazekas score grades 0 and 1 , relative CBF $<25 \%$ for periventricular hyperintensity Fazekas score 2, and relative CBF $<20 \%$ for periventricular hyperintensity Fazekas score 3. The reliability analysis according to periventricular hypodensity Fazekas score grades on NCCT was similar to that in follow-up MR imaging.

CONCLUSIONS: In patients with stroke, the presence of leukoaraiosis confounds the interpretation of CTP despite proper adjustment of CBF thresholds.

ABBREVIATIONS: $\mathrm{FIV}=$ final infarct volume; ICC = intraclass correlation coefficient; $\mathrm{IQR}=$ interquartile range; $\mathrm{MT}=$ mechanical thrombectomy; $\mathrm{NVT}=$ nonviable tissue; $\mathrm{PVH}=$ periventricular hyperintensity/hypodensity Fazekas score; $\mathrm{rCBF}=$ relative $\mathrm{CBF}$

$\mathbf{T}$ he main utility of perfusion images obtained from patients with acute ischemic stroke is the identification and quantifi-

Received March 22, 2019; accepted after revision June 19.

From the Department of Neuroscience (S.R., C.L., X.U., L.L., A.R., V.O., Y.Z., S.A., Á.C.)., Comprehensive Stroke Center, Hospital Clinic, University of Barcelona and August Pi i Sunyer Biomedical Research Institute (IDIBAPS), Barcelona, Spain; Neurosurgery Service (C.V.), Universitary Hospital La Paz, Madrid, Spain; Medicine Department (Á.C.), School of Medicine, University of Barcelona, Barcelona, Spain; and Department of Radiology (J.L.M., A.L.-R.), Hospital Clínic, University of Barcelona, Barcelona, Spain.

Salvatore Rudilosso and Carlos Laredo contributed equally to this work.

Salvatore Rudilosso receives funding from Instituto de Salud Carlos III, with a Grant for Health Research (CM18/00116). Carlos Laredo receives funding from Instituto de Salud Carlos III, with a Predoctoral Grant for Health Research (PFIS, FI16/ 00231). Yashu Zhao received a scholarship from China Scholarship Council. This work was partially developed at the building Centro Esther Koplowitz, Barcelona, Research Centers of Catalonia (CERCA) Programme/Generalitat de Catalunya. cation of areas of nonviable tissue (NVT) and areas of ischemic penumbra that represent hypoperfused but still salvageable tissue. ${ }^{1,2}$ These focal alterations in patients eligible for acute stroke therapies can be evaluated by dynamic contrast-enhanced CT and MR imaging acquisitions, ${ }^{3,4}$ the former being the most commonly used technique worldwide for the assessment of cerebral perfusion in patients with acute stroke. ${ }^{5}$ However, a great variability

Please address correspondence to Ángel Chamorro and Sergio Amaro, Comprehensive Stroke Center, Department of Neuroscience, Hospital Clinic, Villarroel 170, 08036 Barcelona, Spain; e-mail: achamorro@clinic.ub.es and samaro@clinic.cat; @ictusclinic

- Indicates open access to non-subscribers at www.ajnr.org

$\equiv$ Indicates article with supplemental on-line tables.

Indicates article with supplemental on-line photo.

http://dx.doi.org/10.3174/ajnr.A6139 
exists not only in image acquisition and postprocessing techniques but also in intrinsic features of cerebral perfusion. ${ }^{6}$ In fact, in physiologic conditions and in acute focal ischemia, brain perfusion is not the same in all its areas because it depends on the neuronal activity of different regions, ${ }^{7,8}$ where perfusion patterns may be different in patients with similar sites of occlusion and times from stroke onset to imaging acquisition. ${ }^{9}$ This variability in perfusion features may depend mostly on the efficiency of intrinsic compensatory mechanisms such as the collateral blood supply ${ }^{10}$ or vasodilation and oxygen extraction at capillary levels. ${ }^{11}$ Moreover, brain perfusion might also depend on the extent of leukoaraiosis, which is an age-related pathologic process strongly associated with cardiovascular risk factors and stroke. ${ }^{12}$ A recent study conducted on a large cohort of patients with acute stroke showed that hypoperfusion in the periventricular areas was related to the grade of leukoaraiosis, but a quantitative analysis was not assessed. ${ }^{13}$

Therefore, leukoaraiosis may be associated with hypoperfusion and may cause an overestimation of the NVT in ischemic stroke, confounding the interpretation of CTP in patients eligible for reperfusion therapy. In this study, we aimed to quantify the cerebral perfusion parameters according to the grade of leukoaraiosis in a cohort of patients with large-vessel stroke treated with mechanical thrombectomy (MT) and to assess the accuracy of the estimation of infarct core based on variable CBF thresholds.

\section{MATERIALS AND METHODS}

\section{Patients}

This is a retrospective single-center study of 236 patients with large-vessel ischemic stroke in the anterior circulation treated with MT within 3.9 hours (interquartile range [IQR], 2.6-6.0 hours) from stroke onset who had pretreatment multimodal CT imaging and brain MR imaging at follow-up from March 2010 to December 2017 (Fig 1). Reperfusion after MT was quantified with the modified TICI scale. ${ }^{14}$

The local Clinical Research Ethics Committee from the Hospital Clínic of Barcelona approved the study protocol (HCB/ 2019/0077) under the requirements of Spanish legislation in the field of biomedical research, the regulation (European Union) 2016/679 of the European Parliament and of the Council of April 27,2016 on the protection of persons with regard to the processing of personal data and on the free movement of such data, the standards of Good Clinical Practice, as well as with the Helsinki Declaration of 1975 and 1983. Patient consent was not required due to the retrospective nature of the study design.

\section{Neuroimaging Techniques}

Multimodal imaging included, on admission, an NCCT, an angio-CT of the cervical and intracranial arteries, and a CTP. CTP was performed within a median of 166 minutes (IQR, 88-265 minutes) of stroke onset with a Somatom Definition Flash 128-section dualsource CT system (Siemens, Erlangen, Germany) with a $98-\mathrm{mm} \mathrm{z-}$ coverage and 31 time points (total acquisition time, 60 seconds); 50 $\mathrm{mL}$ of nonionic iodinated contrast was administered intravenously at $5 \mathrm{~mL} / \mathrm{s}$ using a power injector. CTP imaging parameters were 80 $\mathrm{kV}$ (peak), $250 \mathrm{mAs}$, 1.5-second rotation, and 2-mm thickness. CTP maps were calculated with MIstar (Apollo Medical Imaging Technology, Melbourne, Australia), which uses singular-value decompo-

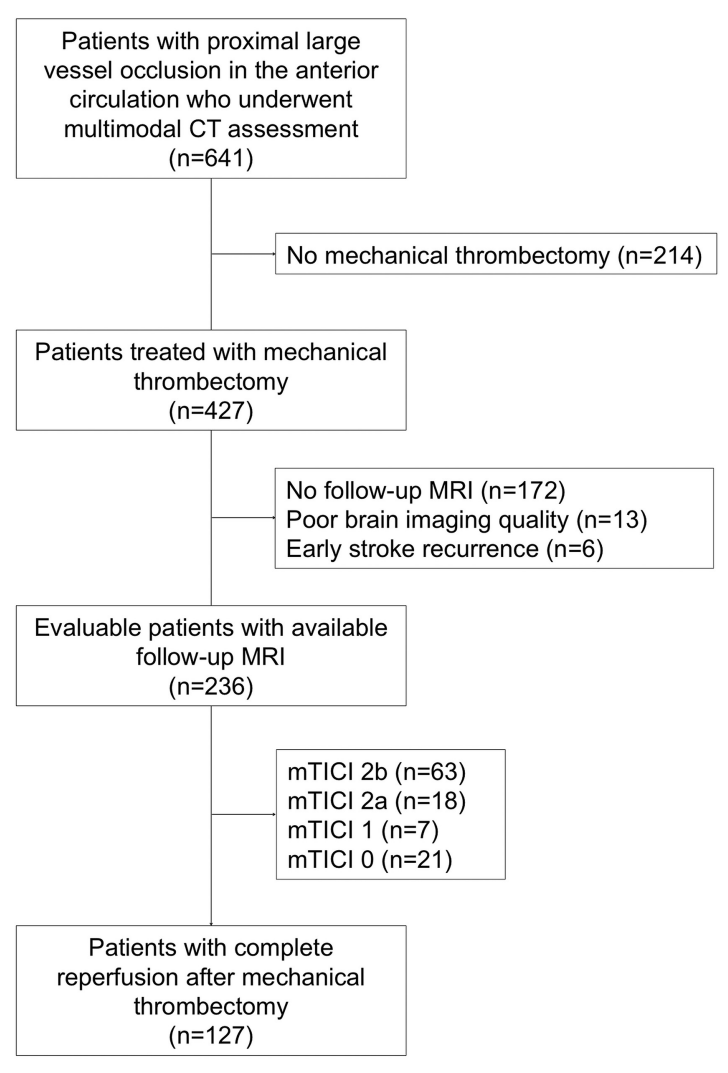

FIG 1. Flow chart of the study cohort.

sition without delay correction and automatically performs motion correction and selects an arterial input function from an unaffected artery and venous output function from a large draining vein. We generated the following perfusion maps: MTT, CBV, and CBF. The NVT was segmented automatically on the CBF maps and defined as a relative threshold of $\mathrm{CBF}$ ( $\mathrm{rCBF})<30 \%$ of the unaffected whole contralateral hemisphere.

MR imaging was performed at a median of 31 hours (IQR, 21-62 hours) after multimodal CT on a $1.5 \mathrm{~T}$ scanner and included a DWI sequence obtained with b-values of 0 and 1000 , 5 -mm section thickness, and $128 \times 128$ matrix. FLAIR images were obtained with $\mathrm{TR}=9000 \mathrm{~ms}, \mathrm{TE}=114 \mathrm{~ms}, \mathrm{FOV}=240$ $\mathrm{mm}$, and slice thickness $=5 \mathrm{~mm}$. The final infarct volume (FIV) was calculated with a semiautomatic segmentation of the acute ischemic lesion on DWI sequences using Amira software (www.amira.com) as previously described. ${ }^{15}$

\section{Neuroimaging Analysis in the Whole Cohort}

Analyzing independently NCCT and FLAIR MR imaging sequences, 1 trained investigator (S.R.) used the Fazekas scale ${ }^{16}$ to calculate the extent of leukoaraiosis in the asymptomatic hemisphere only, thus avoiding the confounding effect of acute ischemic lesions. According to the neuroimaging technique, white matter changes were evaluated as periventricular hyperintensities on MR imaging and periventricular hypodensities on NCCT (periventricular hyperintensity/hypodensity Fazekas score $[\mathrm{PVH}])$. In accordance with this scale, grade 0 defined the absence of white matter changes, grade 1 included caps or pencil-thin lining, grade 2 defined a smooth halo, and 
Table 1: General clinical and radiologic characteristics of the whole population and in patients with complete reperfusion

\begin{tabular}{|c|c|c|}
\hline & $\begin{array}{l}\text { Whole Cohort } \\
\qquad(n=236)\end{array}$ & $\begin{array}{l}\text { mTICI } 3 \text { Subgroup } \\
\quad(n=127)\end{array}$ \\
\hline Age (mean) (SD) (yr) & $68.7(14.6)$ & $68.8(14.2)$ \\
\hline Female sex (No.) (\%) & $116(49.2)$ & $62(49.2)$ \\
\hline Baseline NIHSS score (median) (IQR) & $11(17-21)$ & $16(9-20)$ \\
\hline Hypertension (No.) (\%) & $132(55.9)$ & $68(53.5)$ \\
\hline Diabetes mellitus (No.) (\%) & $34(14.4)$ & $21(16.5)$ \\
\hline Atrial fibrillation (No.) (\%) & $70(29.7)$ & $37(29.1)$ \\
\hline Previous stroke (No.) (\%) & $22(9.3)$ & $11(8.7)$ \\
\hline $\begin{array}{l}\text { Time from symptom onset to CTP study } \\
\text { (median) (IQR) (min) }\end{array}$ & $167(88-270)$ & $150(77-281)$ \\
\hline $\begin{array}{l}\text { Time from CTP to MRI study (median) } \\
\text { (IQR) (hr) }\end{array}$ & $34.9(22.5-61.3)$ & $30.9(20.9-62.2)$ \\
\hline \multicolumn{3}{|l|}{ TOAST (No.) $(\%)^{33}$} \\
\hline Atherosclerotic & $35(14.8)$ & $22(12.7)$ \\
\hline Cardioembolic & $120(50.8)$ & $63(49.6)$ \\
\hline Undetermined & $67(28.4)$ & $36(28.3)$ \\
\hline Infrequent & $14(6)$ & $6(4.7)$ \\
\hline \multicolumn{3}{|l|}{ mTICl score (No.) (\%) } \\
\hline 0 & $21(8.9)$ & $0(0)$ \\
\hline 1 & $7(3)$ & $0(0)$ \\
\hline $2 a$ & $18(7.6)$ & $0(0)$ \\
\hline $2 b$ & $63(26.7)$ & $0(0)$ \\
\hline 3 & $127(53.8)$ & $127(100)$ \\
\hline Collateral status (median) (IQR) & $2(1-3)$ & $2(2-3)$ \\
\hline $\mathrm{CBF}$ (median) (IQR) (mL/100 g/min) & $2.4(2.2-2.6)$ & $2.4(2.2-2.6)$ \\
\hline CBV (median) (IQR) (mL/100 g/min) & $24.3(20.5-26.9)$ & $24.3(20.5-26.5)$ \\
\hline MTT (median) (IQR) (sec) & $6.0(5.5-6.5)$ & $6.1(5.5-6.6)$ \\
\hline NVT (median) (IQR) (mL) & $19.3(8.0-34.5)$ & $16.0(7.4-33.0)$ \\
\hline FIV (median) (IQR) (mL) & $19.6(7.4-51.0)$ & $14.0(3.6-58.4)$ \\
\hline
\end{tabular}

Note:- mTICI indicates modified TICI.

Whole cohort $(n=236)$

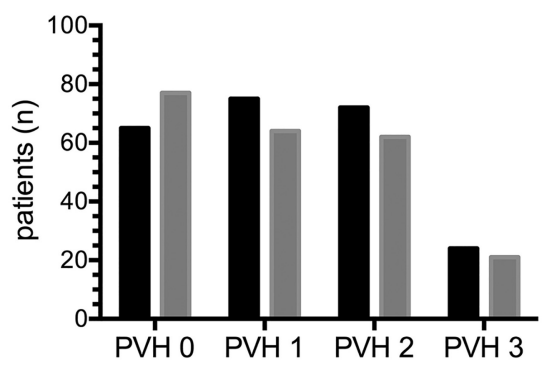

Periventricular Fazekas score

MRI evaluation

NCCT evaluation

FIG 2. PVHs on MR imaging and noncontrast $\mathrm{CT}$.

grade 3 defined irregular periventricular lesions extending into the deep white matter.

\section{Neuroimaging Analysis in Patients with Complete Reperfusion}

The relationship between the grade of leukoaraiosis and the FIV was analyzed only in patients with complete reperfusion after MT (modified TICI 3) to reduce the confounding effect of variable infarct growth in patients with incomplete reperfusion after MT. In this subgroup, we assessed the intraclass correlation coefficient (ICC) and the volumetric bias between the NVT and the FIV according to the PVH on MR imaging and also explored ICC values across different $\mathrm{rCBF}$ thresholds ( $\mathrm{rCBF}<30 \%$, $25 \%, 20 \%$, and $15 \%$ ). Then, to assess the usefulness of evaluating the grade of leukoaraiosis on the pretreatment imaging, the same reliability analyses were conducted using the subgroups based on the NCCT-derived PVHs. Finally, to assess the internal validity of the study, we repeated the main imaging analysis in patients with complete reperfusion using a different software package (syngo.via CT Neuro Perfusion VB30; Siemens). We calculated the ICC and the volumetric bias according to the PVH on MR imaging and different $\mathrm{rCBF}$ thresholds ( $\mathrm{rCBF}<30 \%$ and $20 \%$ ).

\section{Statistics}

Categoric variables were compared using the $\chi^{2}$ test if suitable, and alternatively using the Fisher exact test. Continuous variables with normal distribution were described by mean and SD, while continuous variables without normal distribution and ordinal variables were described by median and IQR. Means between 2 groups were compared using the Student $t$ test, and medians between 2 groups were compared using the Mann-Whitney $U$ or Kruskal-Wallis test as appropriate. The agreement between MR imaging and NCCT PVHs was evaluated with a linear-weighted $\kappa$ statistic. The relationship between the MTT, $\mathrm{CBV}$, and MTT values in the asymptomatic hemisphere with the Fazekas score was evaluated using the Spearman $\rho$ correlation coefficient. The ICC was interpreted as previously described (ICC < 0.4 , poor reliability; ICC, $0.4-0.75$, from fair-to-good reliability; ICC > 0.75 , excellent reliability). ${ }^{17}$ Volumetric bias was defined as the difference between the predicted and final infarct (NVT-FIV) across PVH groups. All statistical analyses were performed using SPSS 23.0 (IBM, Armonk, New York). We considered as statistically significant $P$ values $<.05$, and all hypotheses were 2 -sided.

\section{RESULTS}

\section{Leukoaraiosis and Hemodynamic Correlates in the Whole Population}

The main demographic, clinical, and radiologic features of the whole study cohort $(n=236)$ and of patients with complete reperfusion at the end of MT $(n=127)$ are shown in Table 1. PVHs of the whole cohort evaluated on posttreatment MR imaging and pretreatment NCCT are shown in Fig 2. Overall, the agreement 


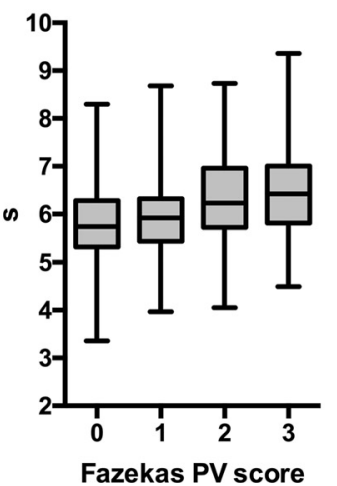

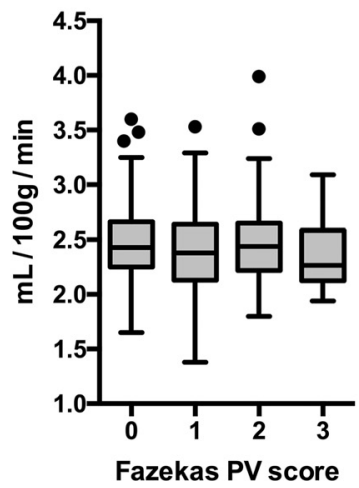

FIG 3. Boxplot analysis of CTP maps in the asymptomatic hemisphere according to the PVH score on MR imaging in the whole population $(N=236)$.

PVH 0

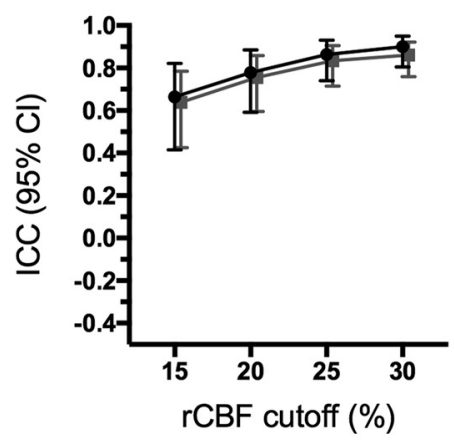

PVH 2

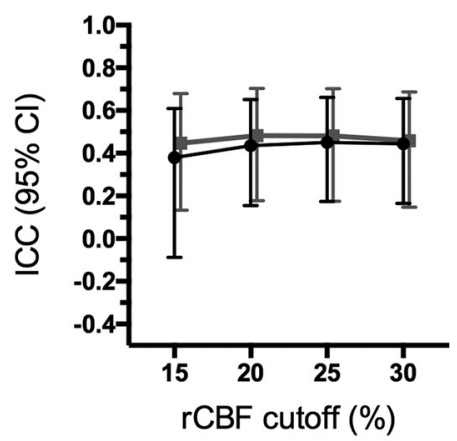

$\mathrm{PVH} 1$

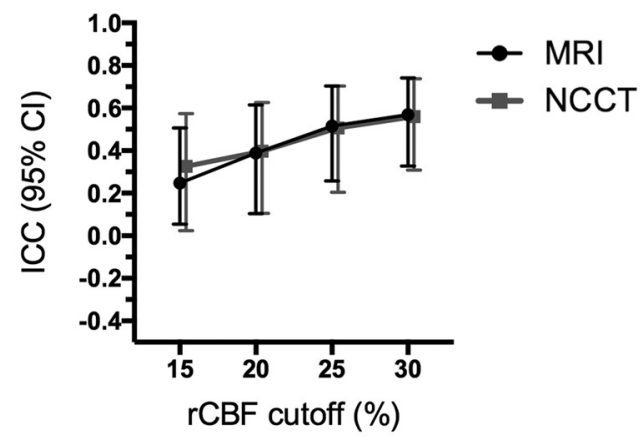

$\mathrm{PVH} 3$

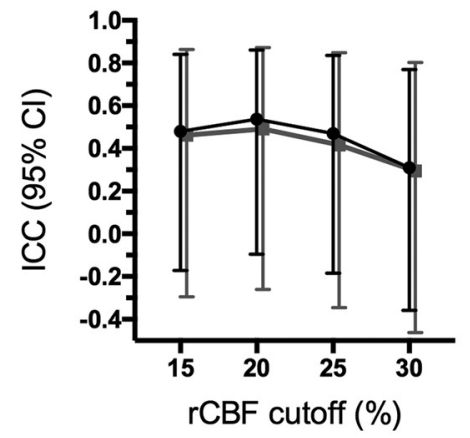

FIG 4. ICC analysis of the predicted final infarct volume on CTP according to different rCBF cutoffs and the grade of leukoaraiosis assessed by the PVH on follow-up MR imaging and on pretreatment NCCT in the subgroup of patients with complete reperfusion after mechanical thrombectomy ( $n=127)$. Of note, the agreement in patients without leukoaraiosis was excellent for $\mathrm{rCBF}<30 \%$ and $\mathrm{rCBF}<25 \%$ cutoffs and remained good for $\mathrm{rCBF}<20 \%$ and $\mathrm{rCBF}<15 \%$. In patients with leukoaraiosis, across increasing PVHs, the overall reliability of CTP decreased and more restrictive thresholds (mostly $\mathrm{rCBF}<25 \%$ and $\mathrm{rCBF}<20 \%$ ) had better agreement than the commonly used $\mathrm{rCBF}<30 \%$.

between the evaluations on MR imaging and NCCT was good $(\kappa=0.69 ; 95 \%$ CI, 0.62-0.76). Higher PVHs were associated with longer MTT (Spearman $\rho=0.279, P<.001)$ and lower CBF $(\rho=$ $-0.263, P<.001)$ but were not correlated with CBV values $(\rho=$ $-0.043, P=.513$ ), as shown in Fig 3 and On-line Table 1 . There was no correlation between CBF, CBV, and MTT values in the asymptomatic hemisphere and $\operatorname{NVT}(P=.573, P=.251$, and $P=$ .978 , respectively) or FIV $(P=.902, P=.207$, and $P=.522$, respectively). NVT was similar in patients with different grades of reperfusion $(P=.657)$, but the FIV was inversely related to TICI subgroups ( $\rho=$ $-0.450, P<.001)$.

CTP Prediction of FIV in Relation to the Extent of Leukoaraiosis in Patients with Complete Reperfusion after MT

In patients with complete reperfusion at the end of MT ( $n=127)$, the agreement between NVT and FIV using the rCBF $<30 \%$ cutoff was inversely correlated with PVH $(\rho=-0.199, P<.001)$ : ICC was excellent (ICC, 0.900; 95\% CI, 0.805-0.950) for patients with PVH 0 graded on MR imaging, remained fair for patients with PVH 1 (ICC, 0.569; 95\% CI, 0.327-0.741) and PVH 2 (ICC, 0.444; 95\% CI, 0.165-0.657), and was poor for patients with PVH 3 (ICC, $0.310 ; 95 \%$ CI, $-0.359-0.769)$. The analysis of CTP prediction of the FIV based on the NCCT PVH evaluation showed results similar to those of the MR imaging-based analysis as shown in Fig 4 and in On-line Table 2. Median volumetric bias was similar among MR imaging-derived PVH subgroups regardless of the technique used to grade leukoaraiosis (Table 2). Two representative cases illustrating these observations are shown in Fig 5.

A comprehensive threshold-finding analysis across different CTP-derived rCBF cutoffs was implemented to evaluate whether the extent of leukoaraiosis affected the prediction of FIV. In this exploratory analysis, the $\mathrm{rCBF}<30 \%$ threshold showed the highest ICC between NVT and FIV in patients with PVHs 0 and 1, whereas the $\mathrm{rCBF}<25 \%$ and $\mathrm{rCBF}<20 \%$ thresholds had a higher ICC than $\mathrm{rCBF}<30 \%$ for patients with PVHs 2 and 3, regardless of the neuroimaging technique used for the evaluation of leukoaraiosis (Fig 4). The $\mathrm{rCBF}<15 \%$ cutoff was less reliable for each grade of leukoaraiosis. The correlation between NVT-FIV ICC and $\mathrm{PVH}$ was less strong for $\mathrm{rCBF}<25 \%(\rho=-0.153, P=.002)$ and $\mathrm{rCBF}<20 \%(\rho=-0.129, P=.01)$ thresholds compared with the $\mathrm{rCBF}<30 \%$ threshold, while no significant correlation was found for the rCBF $15 \%$ threshold $(\rho=-0.074, P=$ .139). Complete data of this analysis are shown in the On-line Table 2. Of note, the distribution of collateral scores and time to recanalization was not different among $\mathrm{PVH}$ categories as shown in On-line Table 3.

In the comparative analysis of different commercial software 
Table 2: Baseline traits and volumetric differences among PVH scores on MRI in patients achieving complete reperfusion after mechanical thrombectomy $(n=126)$

\begin{tabular}{|c|c|c|c|c|c|}
\hline & PVH 0 & PVH 1 & PVH 2 & PVH 3 & $P$ Value \\
\hline Age (mean) (SD) (yr) & $59.0(16.7)$ & $68.0(12.2)$ & $76.6(7.7)$ & $72.9(15.3)$ & $<.001$ \\
\hline Baseline NIHSS score (median) (IQR) & $16(11-19)$ & $16(11-20)$ & $18(8-20)$ & $17(13-21)$ & .921 \\
\hline Time from symptom onset to CTP study (median) (IQR) (min) & $154(89-233)$ & $168(92-296)$ & $135(64-263)$ & $185(73-385)$ & .871 \\
\hline $\mathrm{CBF}$ (median) (IQR) (mL/100 g/min) & $2.4(2.2-2.7)$ & $2.4(2.1-2.6)$ & $2.5(2.2-2.7)$ & $2.4(2.2-2.8)$ & .558 \\
\hline CBV (median) (IQR) (mL/100 g/min) & $25.4(22.9-28.1)$ & $23.9(21.0-26.1)$ & $23.1(19.4-26.9)$ & $22.6(18.0-28.0)$ & .205 \\
\hline MTT (median) (IQR) (sec) & $5.7(5.3-6.3)$ & $6.0(5.4-6.3)$ & $6.2(5.8-6.7)$ & $6.4(6.3-7.1)$ & .021 \\
\hline Collateral status (median) (IQR) & $2(2-3)$ & $2(2-3)$ & $2(2-3)$ & $2(1-2)$ & .174 \\
\hline NVT (median) (IQR) (mL) & $15.4(7.3-32.0)$ & $16.0(7.8-36.3)$ & $15.4(3.5-31.4)$ & $23.1(15.1-33.9)$ & .411 \\
\hline FIV (median) (IQR) (mL) & $15.0(3.5-32.4)$ & $12.8(5.3-19.8)$ & $14.3(2.8-40.0)$ & $13.6(1.3-25.8)$ & .826 \\
\hline Volumetric bias ${ }^{\mathrm{a}}$ (median) (IQR) (mL) using Fazekas score on MRI & $4.2(-18.5-9.8)$ & $1.6(-3.1-16.4)$ & $0.0(-18.7-16.0)$ & $12.5(0.9-30.4)$ & .205 \\
\hline Volumetric bias ${ }^{\mathrm{a}}$ (median) (IQR) (mL) using Fazekas score on NCCT & $2.6(-12.1-11.6)$ & $2.3(-3.5-13.2)$ & $0.5(-18.7-14.5)$ & $11.4(-1.1-31.4)$ & .557 \\
\hline
\end{tabular}

a Volumetric bias was defined as NVT (using the rCBF $<30 \%$ cutoff)-FIV.

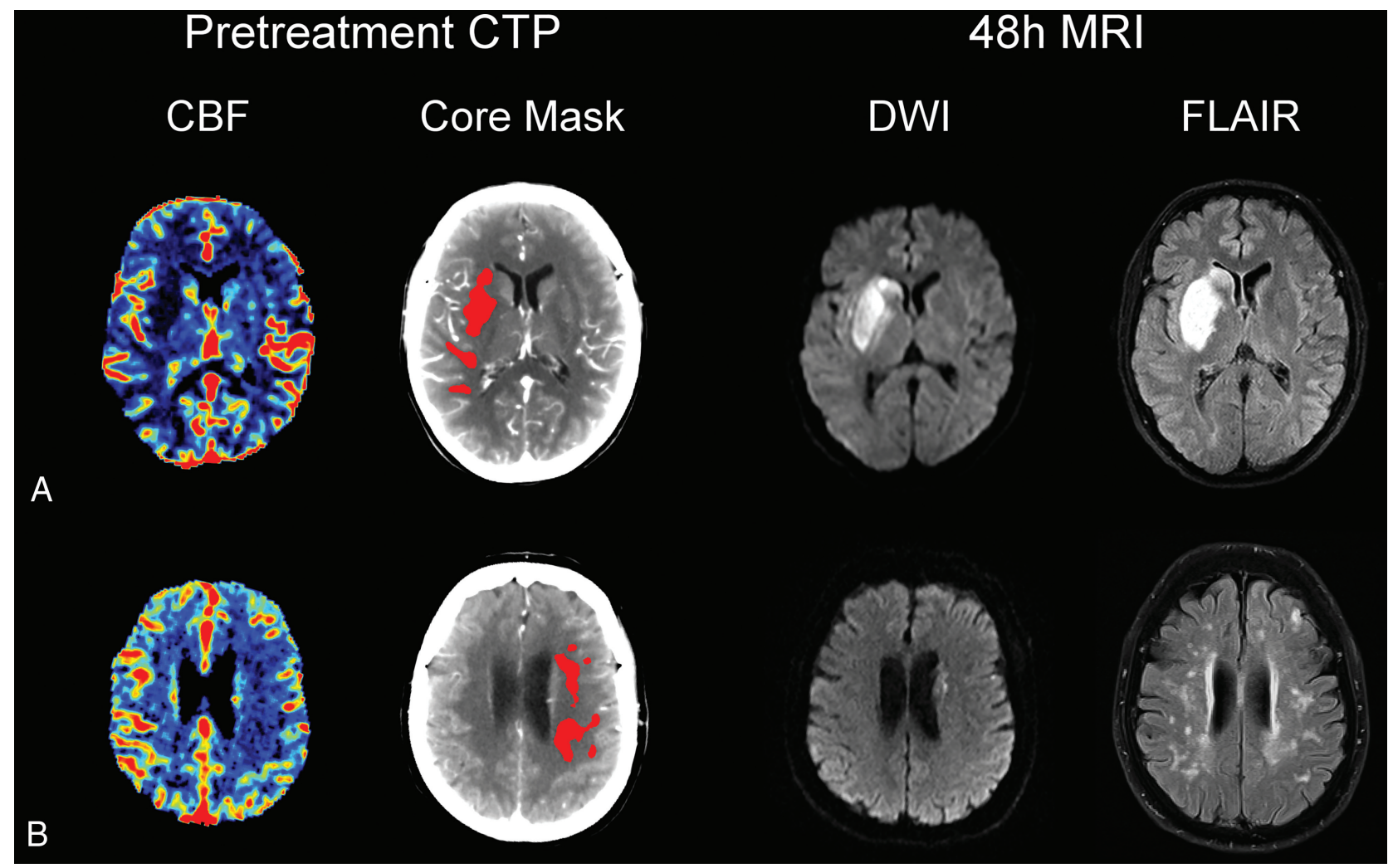

FIG 5. Representative cases of patients with acute ischemic stroke treated with mechanical thrombectomy. A, A 38-year-old patient with low-grade leukoaraiosis (PVH 0). B, A 79-year-old patient with high-grade leukoaraiosis (PVH 3). Both patients had a left middle cerebral artery stroke due to $\mathrm{Ml}$ occlusion and had complete reperfusion (modified TICI score of 3 ) after mechanical thrombectomy. The CTP-predicted NVT on CBF was similar to final infarct volume in patient $\mathrm{A}$ (NVT $=17.9 \mathrm{~mL}$, FIV $=22.2 \mathrm{~mL}$ ), while in patient $\mathrm{B}, \mathrm{CTP}$-predicted NVT overestimated the FIV (NVT $=11.2 \mathrm{~mL}$, FIV $=0.8 \mathrm{~mL}$ ).

packages, the agreement of NVT estimation using the rCBF $<30 \%$ threshold between MIstar and syngo.via software was good (ICC, 0.664; 95\% CI, 0.469-0.782). A representative example is shown in the On-line Figure. With syngo.via, there was still an inverse correlation with PVHs for both $\mathrm{rCBF}<30 \%(\rho=-0.275$, $P<.001)$ and $\mathrm{rCBF}<20 \%(\rho=-0.119, P=.018)$. The $\mathrm{rCBF}$ $<20 \%$ cutoff showed a higher ICC compared with $\mathrm{rCBF}<30 \%$ in patients with $\mathrm{PVH}>0$ as shown in On-line Table 4.

\section{DISCUSSION}

In this study, we investigated whether the presence and severity of leukoaraiosis confounded the interpretation of CTP findings for the prediction of final infarct volume in patients with complete reperfusion following MT. The main result of the study was that the extent of leukoaraiosis altered the prediction of final infarct volume following MT but was not correlated to overestimation of the final infarct volume. Although the use of more restrictive rCBF thresholds may improve the estimation of tissue viability in patients with a high grade of leukoaraiosis, the accuracy of CTP was reduced in these patients. Finally, the study also found that NCCT was a reliable imaging technique to identify those patients whose extent of leukoaraiosis could compromise the accuracy of CTP interpretation.

The estimation of infarct core by means of a relative $\mathrm{CBF}$ threshold lower than $30 \%(\mathrm{rCBF}<30 \%)$ is broadly accepted to define NVT in acute stroke. ${ }^{18-20}$ In our study, the reliability of the rCBF $<30 \%$ threshold decreased in relation to an increased 
prominence of leukoaraiosis. Thus, the $\mathrm{rCBF}<30 \%$ threshold was only excellent for the prediction of FIV in patients without leukoaraiosis (PVH 0). Conversely, in patients with mild (PVH 1) and moderate (PVH 2) leukoaraiosis, the application of that threshold had a lower but still fair grade of agreement between CTP-derived NVT and the FIV on follow-up MR imaging, while tissue-viability prediction was only seriously hampered in patients with a severe grade of leukoaraiosis (PVH 3). According to these results, the $\mathrm{rCBF}<30 \%$ cutoff remained the most reliable in patients with PVHs 0 and 1. However, in patients with higher grades of leukoaraiosis, the prediction of tissue viability was only moderate, even after applying more restrictive thresholds ( $\mathrm{rCBF}$ $<25 \%$ or rCBF $<20 \%$ cutoffs). Therefore, the burden of leukoaraiosis did not reflect overestimation of the infarct core despite an insignificant tendency for higher volumetric bias in the PVH 3 subgroup, and the worse FIV prediction might also depend on other factors that may affect hemodynamic measures in patients with small-vessel disease, such as blood-brain barrier permeability derangements, ${ }^{21,22}$ collateral circulation status, ${ }^{23}$ and cerebrovascular reactivity. ${ }^{24,25}$

In agreement with previous studies, the evaluation of periventricular leukoaraiosis on NCCT scans showed a good grade of agreement with the evaluation of the Fazekas scale on MR imaging. ${ }^{26,27}$ Accordingly, our study supports the use of NCCT to quantify the grade of leukoaraiosis and thus to improve the accuracy of the interpretation of CTP maps in potential candidates for endovascular therapy, especially in extended time windows in which the presence of severely hypoperfused areas might preclude the use of reperfusion therapy according to recent randomized clinical trials. ${ }^{28-30}$ Although perfusion imaging adds useful data about tissue viability in stroke assessment, our results highlighted the limitations of CTP in the prediction of final infarct, and support the current stroke guideline indications based on NCCT ASPECTS evaluation for endovascular therapies in the first 6-hour window, while perfusion imaging is required only in extended time windows. Further studies are warranted to assess the role of leukoaraiosis on the accuracy of CTP maps in patients with stroke imaged in late time windows.

The main results of this study were confirmed by using alternative commercial software packages. Despite small differences related to optimal thresholds according to the software used, ${ }^{31}$ the accuracy of final infarct prediction was still inversely related to the grade of leukoaraiosis, and the more restrictive $\mathrm{rCBF}<20 \%$ cutoffs were shown to be more suitable in patients with a high-grade of white matter hyperintensities as well.

This study has some limitations. First, the time from CTP to follow-up MR imaging may affect the accuracy of the FIV prediction due to possible dynamic alterations in the DWI intensity. ${ }^{32}$ However, our cohort was rather homogeneous because most patients had undergone MR imaging within 48 hours from MT. Second, the low prevalence of patients with PVH 3 in this cohort and the lack of a specific assessment of perfusion parameters in the white matter and the mirror region of the final infarct could have underpowered the analysis of CTP maps. Nevertheless, this cohort study represented faithfully the population eligible for stroke endovascular therapy at our center, and these findings may thus be valid for regular use in clinical practice.

\section{CONCLUSIONS}

The extent of leukoaraiosis in patients with acute stroke eligible for MT impairs the accuracy of CTP maps for the prediction of tissue viability, even despite appropriate adjustment of CBF cutoffs. Therefore, the variable prevalence of leukoaraiosis in this clinical setting should be accounted for at the time of the perfusion study evaluation. Further studies assessing the characteristics of white matter hemodynamics in acute stroke are required to provide further insight into the efficacy of reperfusion therapies in patients with this condition.

Disclosures: We thank the support of the Spanish Ministry of Economy and Competitiveness for grant to Sergio Amaro (project PI16/00711 funded by Instituto de Salud Carlos III and co-funded by European Regional Developement Fund [ERDF]).

\section{REFERENCES}

1. Donnan GA, Davis SM. Neuroimaging, the ischaemic penumbra, and selection of patients for acute stroke therapy. Lancet Neurol 2002;1:417-25 CrossRef Medline

2. Dani KA, Thomas RG, Chappell FM, et al; Translational Medicine Research Collaboration Multicentre Acute Stroke Imaging Study. Computed tomography and magnetic resonance perfusion imaging in ischemic stroke: definitions and thresholds. Ann Neurol 2011; 70:384-401 CrossRef Medline

3. Etherton MR, Barreto AD, Schwamm LH, et al. Neuroimaging paradigms to identify patients for reperfusion therapy in stroke of unknown onset. Front Neurol 2018;9:327 CrossRef Medline

4. Vilela P, Rowley HA. Brain ischemia: CT and MRI techniques in acute ischemic stroke. Eur J Radiol 2017;96:162-72 CrossRef Medline

5. Donahue J, Wintermark M. Perfusion CT and acute stroke imaging: foundations, applications, and literature review. J Neuroradiol 2015;42:21-29 CrossRef Medline

6. Heit JJ, Wintermark M. Perfusion computed tomography for the evaluation of acute ischemic stroke: strengths and pitfalls. Stroke 2016;47:1153-58 CrossRef Medline

7. Ramli N, Ho K, Nawawi O, et al. CT perfusion as a useful tool in the evaluation of leuko-araiosis. Biomed Imaging Interv J 2006;2:e16 CrossRef Medline

8. Chen C, Bivard A, Lin L, et al. Thresholds for infarction vary between gray matter and white matter in acute ischemic stroke: a CT perfusion study. J Cereb Blood Flow Metab 2019;39:536-46 CrossRef Medline

9. Sakai Y, Delman BN, Fifi JT, et al. Estimation of ischemic core volume using computed tomographic perfusion. Stroke 2018;49: 2345-52 CrossRef Medline

10. Vagal A, Aviv R, Sucharew H, et al. Collateral clock is more important than time clock for tissue fate. Stroke 2018;49:2102-07 CrossRef Medline

11. Østergaard L, Jespersen SN, Mouridsen K, et al. The role of the cerebral capillaries in acute ischemic stroke: the extended penumbra model. J Cereb Blood Flow Metab 2013;33:635-48 CrossRef Medline

12. Higashida RT, Furlan AJ, Roberts H, et al; Technology Assessment Committee of the American Society of Interventional and Therapeutic Neuroradiology, Technology Assessment Committee of the Society of Interventional Radiology. Trial design and reporting standards for intra-arterial cerebral thrombolysis for acute ischemic stroke. Stroke 2003;34:e109-37 Medline

13. Arba F, Mair G, Carpenter T, et al; IST-3 Trial Collaborators. Cerebral white matter hypoperfusion increases with small-vessel disease burden: data from the Third International Stroke Trial. J Stroke Cerebrovasc Dis 2017;26:1506-13 CrossRef Medline

14. Zaidat OO, Lazzaro MA, Liebeskind DS, et al. Revascularization grading in endovascular acute ischemic stroke therapy. Neurology 2012;79:S110-16 CrossRef Medline

15. Rudilosso S, Urra X, San Román L, et al. Perfusion deficits and mis- 
match in patients with acute lacunar infarcts studied with wholebrain CT perfusion. AJNR Am J Neuroradiol 2015;36:1407-12 CrossRef Medline

16. Fazekas F, Chawluk JB, Alavi A, et al. MR signal abnormalities at $\mathbf{1 . 5}$ $T$ in Alzheimer's dementia and normal aging. AJR Am J Roentgenol 1987;149:351-56 CrossRef Medline

17. Rosner B. Fundamentals of Biostatistics. 6th ed. Thomson: Brooks/ Cole; 2006

18. Yu Y, Han Q, Ding X, et al. Defining core and penumbra in ischemic stroke: a voxel- and volume-based analysis of whole brain CT perfusion. Sci Rep 2016;6:20932 CrossRef Medline

19. Lin L, Bivard A, Krishnamurthy V, et al. Whole-brain CT perfusion to quantify acute ischemic penumbra and core. Radiology 2016;279: 876-87 CrossRef Medline

20. Campbell BC, Christensen S, Levi CR, et al. Cerebral blood flow is the optimal CT perfusion parameter for assessing infarct core. Stroke 2011;42:3435-40 CrossRef Medline

21. Wardlaw JM, Smith C, Dichgans M. Small vessel disease: mechanisms and clinical implications. Lancet Neurol 2019;18:684-96 CrossRef Medline

22. Wardlaw JM, Doubal FN, Valdes-Hernandez M, et al. Blood-brain barrier permeability and long-term clinical and imaging outcomes in cerebral small vessel disease. Stroke 2013;44:525-27 CrossRef Medline

23. Giurgiutiu DV, Yoo AJ, Fitzpatrick K, et al. Severity of leukoaraiosis, leptomeningeal collaterals, and clinical outcomes after intra-arterial therapy in patients with acute ischemic stroke. J Neurointerv Surg 2015;7:326-30 CrossRef Medline

24. Shi Y, Thrippleton MJ, Blair GW, et al. Small vessel disease is associated with altered cerebrovascular pulsatility but not resting cerebral blood flow. J Cereb Blood Flow Metab 2018 Oct 8:271678X18803956. [Epub ahead of print] CrossRef Medline

25. Østergaard L, Engedal TS, Moreton F, et al. Cerebral small vessel disease: capillary pathways to stroke and cognitive decline. J Cereb Blood Flow Metab 2016;36:302-25 CrossRef

26. Rudilosso S, San Román L, Blasco J, et al. Evaluation of white matter hypodensities on computed tomography in stroke patients using the Fazekas score. Clin Imaging 2017;46:24-27 CrossRef Medline

27. Ferguson KJ, Cvoro V, MacLullich AM, et al. Visual rating scales of white matter hyperintensities and atrophy: comparison of computed tomography and magnetic resonance imaging. J Stroke Cerebrovasc Dis 2018;27:1815-21 CrossRef Medline

28. Albers GW, Marks MP, Kemp S, et al; DEFUSE 3 Investigators. Thrombectomy for stroke at $\mathbf{6}$ to $\mathbf{1 6}$ hours with selection by perfusion imaging. $N$ Engl J Med 2018;378:708-18 CrossRef Medline

29. Nogueira RG, Jadhav AP, Haussen DC, et al; DAWN Trial Investigators. Thrombectomy 6 to 24 hours after stroke with a mismatch between deficit and infarct. N Engl J Med 2018;378:11-21 CrossRef Medline

30. Thomalla G, Simonsen CZ, Boutitie F, et al; WAKE-UP Investigators. MRI-guided thrombolysis for stroke with unknown time of onset. N Engl J Med 2018;379:611-22 CrossRef Medline

31. Bathla G, Limaye K, Policeni B, et al. Achieving comparable perfusion results across vendors: the next step in standardizing stroke care: a technical report. J Neurointerv Surg 2019 May 22. [Epub ahead of print] CrossRef Medline

32. Laredo C, Renú A, Tudela R, et al. The accuracy of ischemic core perfusion thresholds varies according to time to recanalization in stroke patients treated with mechanical thrombectomy: a comprehensive whole-brain computed tomography perfusion study. J Cereb Blood Flow Metab 2019 Jun 17:271678X19855885. [Epub ahead of print] CrossRef Medline

33. Adams HP Jr, Bendixen BH, Kappelle LJ, et al. Classification of subtype of acute ischemic stroke. Definitions for use in a multicenter clinical trial. TOAST. Trial of Org 10172 in Acute Stroke Treatment. Stroke 1993;24:35-41 CrossRef Medline 